\title{
Dynamic Characterization of Permeabilities and Flows in Microchannels
}

\author{
M. Castro, ${ }^{1}$ M. E. Bravo-Gutiérrez, ${ }^{2}$ A. Hernández-Machado,${ }^{3}$ and E. Corvera Poiré ${ }^{2, *}$ \\ ${ }^{1}$ GISC and Grupo de Dinámica No Lineal (DNL), Escuela Técnica Superior de Ingeniería (ICAI), Universidad Pontificia Comillas, \\ E-28015 Madrid, Spain \\ ${ }^{2}$ Departamento de Física y Química Teórica, Facultad de Química, Universidad Nacional Autónoma de México, \\ México DF 04510, México \\ ${ }^{3}$ Departament ECM, Facultat de Física, Universitat de Barcelona Diagonal 647, E-08028 Barcelona, Spain
} (Received 22 October 2007; published 26 November 2008)

\begin{abstract}
We make an analytical study of the nonsteady flow of Newtonian fluids in microchannels. We consider the slip boundary condition at the solid walls with Navier hypothesis and calculate the dynamic permeability, which gives the system's response to dynamic pressure gradients. We find a scaling relation in the absence of slip that is broken in its presence. We discuss how this might be useful to experimentally determine - by means of microparticle image velocimetry technology—whether slip exists or not in a system, the value of the slip length, and the validity of Navier hypothesis in dynamic situations.
\end{abstract}

DOI: 10.1103/PhysRevLett.101.224501

PACS numbers: 47.61.- $\mathrm{k}, 47.45 . \mathrm{Gx}, 62.60 .+\mathrm{v}$

Introduction. - The ability to manipulate viscous fluids in channels at micrometer scales has motivated a huge interest in different fields ranging from physics to engineering and biology [1-10]. The cooperation between science and technology requires a deeper understanding of the underlying physics in order to achieve the desired control and extend it to small volumes [2,5,6]. One of the most controversial issues in the field of microfluidics concerns the fluid flow in the proximities of a solid wall $[3,4,7,9,11]$.

Whether the fluid slips at a solid wall or not has been questioned since the times of Navier. Experiments with Newtonian fluids [12] at macroscales have traditionally been in good agreement with the no-slip assumption, which states that the tangential velocity of the fluid at the solid wall vanishes. However, at microscopic scales recent experiments seem to indicate that the no-slip condition no longer holds in all cases $[3,7,9]$. The concept of an apparent slip has then arisen in microfluidics. Explanations of the origin of this apparent slip have included the formation of a gaseous film lying between the fluid and the solid, surface wettability, and surface roughness [14-18]. Despite being far from being understood [3,18-20], the apparent slip is theoretically consistent with a slip velocity at the solid walls.

The simplest assumption for the slip velocity (the tangential fluid velocity at the solid wall) is given by Navier hypothesis,

$$
\mathbf{u}_{\text {slip }} \equiv \lambda \dot{\gamma},
$$

where $\dot{\gamma}$ is the local shear rate and $\lambda$ the so-called slip length, which according to experiments ranges from tens of nanometers to microns $[7,18]$. This equation is the boundary condition at the solid walls of the evolution equation for a Newtonian fluid, namely, the Navier-Stokes equation. Equation (1) was originally proposed by Navier with con- stant $\lambda$. However, recent experimental observations $[8,15]$, as well as molecular dynamics simulations [14], suggest that for certain systems (for example with hydrophobic interactions between fluid and solid) $\lambda$ may be a function of the shear rate $\dot{\gamma}$.

Until now, all of the experiments to determine the slip length have worked in stationary conditions, where an excess flow of the order of nanoliters has to be measured. Moreover, the effect that the apparent slip has on the fluid response to time dependent pressure gradients has not been explored in literature. Such situations arise in countless amounts of biological systems, where flow occurs with characteristic frequencies.

In this Letter we analyze the dynamic behavior of a Newtonian fluid that obeys Navier hypothesis with slip length $\lambda$, independent of the shear rate, at the walls of a microchannel. We make an analytical characterization of the dynamic permeability [21], which contains the information of the fluid response to a dynamic pressure gradient. We find a scaling relation in the absence of slip that is broken in its presence. We propose how this can be useful to experimentally determine-by means of dynamic measurements - whether slip exists or not in a system, and the value of the slip length. Furthermore, such measurements provide a way to test the validity of Navier hypothesis in dynamic situations.

Theory.-We consider the flow of a Newtonian fluid in a rectangular microchannel since this is the geometry that experiments normally use in microfluidics. The walls of the microchannel are two flat plates parallel to each other, separated by a distance $2 l$ that is much smaller than the plates' length. We consider channels whose characteristic length $l$ is of the order of tens of microns. As a consequence, the Reynolds number is low enough to neglect the inertial (nonlinear) term. We solve the linearized NavierStokes equation subject to Navier boundary condition. We 
choose the $x$ axis as the flow direction and the $z$ axis perpendicular to the microchannel.

We consider that the system is subjected to a nonstationary pressure gradient $\nabla p$, which is a function of time. In frequency domain, the Navier-Stokes equation can be written as [22]

$$
\frac{d^{2} \hat{v}}{d z^{2}}+\frac{i \omega \rho}{\eta} \hat{v}=\frac{1}{\eta} \frac{d \hat{p}}{d x} .
$$

On the other hand, Navier hypothesis [Eq. (1)] in frequency domain with constant $\lambda$ can be written as

$$
\hat{u}_{\text {slip }}=\left.\mp \lambda \frac{d \hat{v}}{d z}\right|_{ \pm l} .
$$

We solve Eq. (2) subject to the boundary condition given by Eq. (3). We then average over the cross-sectional area of the microchannel and write a generalized Darcy's law in frequency domain as

$$
\langle\hat{v}(\omega)\rangle \equiv-\frac{K(\omega ; l, \lambda)}{\eta} \frac{d \hat{p}}{d x},
$$

where $K(\omega, l, \lambda)$ is the dynamic permeability. Our result for the dynamic permeability of a fluid in a microchannel with slip is

$$
K(\omega, l, \lambda)=-A^{-2}\left(1-\frac{\tan (A l)}{A l[1-A \lambda \tan (A l)]}\right),
$$

where $A=\sqrt{i \omega \rho / \eta}$. We have written explicitly the dependence of $K$ on $\omega, \lambda$, and $l$ to facilitate the analysis. Equation (5) contains the information about the nonstationary response of the fluid in the microchannel. It is a measure of the resistance to flow; for instance, it has been shown [23] that for a pressure gradient consisting only on one mode $\nabla p(t)=\nabla p_{0} \cos \omega_{0} t$, the velocity in time domain averaged over the cross-sectional area is given by

$$
\begin{aligned}
\langle v(t)\rangle= & -\frac{\nabla p_{0}}{\eta}\left[\operatorname{Re} \hat{K}\left(\omega_{0}\right) \cos \left(\omega_{0} t\right)\right. \\
& \left.+\operatorname{Im} \hat{K}\left(\omega_{0}\right) \operatorname{sen}\left(\omega_{0} t\right)\right] .
\end{aligned}
$$

Note that both the real and the imaginary parts of the dynamic permeability contribute to the flow. Note also that the experimental determination of the velocity profiles in time domain provides a way of determining the real and imaginary parts of the permeability by decomposing the average velocity in two parts, one in phase and one out of phase (by a factor of $-\pi / 2$ ) with the applied pressure gradient [24].

Analysis.-We find that in the absence of slip, the dynamic permeabilities for two different plate separations obey a scaling relation that is broken when slip is present. This result might be useful to experimentally determine the absence or presence of slip in a system. Moreover, if slip does exist, this might provide a way to experimentally determine the slip length $\lambda$. We do this by means of the following argument: first of all, we note that in the absence of slip $(\lambda=0), K(\omega, l, 0) / l^{2}$ depends only on the product $A l$ as can be seen from Eq. (5). For a given fluid, this implies that $K(\omega, l, 0) / l^{2}$ depends only on the product $\omega l^{2}$. Hereby we can write the following scaling relation for the dynamic permeabilities of two different experiments, one with plate separation $2 l$ and frequency $\omega$ and another one with plate separation $2 \beta l$ and frequency $\omega / \beta^{2}$ :

$$
\frac{\beta^{2} K(\omega, l, 0)}{K\left(\omega / \beta^{2}, \beta l, 0\right)}=1,
$$

which holds for any arbitrary positive constant $\beta$.

This scaling relation breaks down when $\lambda \neq 0$ due to the nontrivial effect of adding a new length scale to the problem. In other words, there is a new nondimensional number $(\lambda / l)$ known as the Knudsen number [1] that in the presence of slip causes the ratio $\frac{\beta^{2} K(\omega, l, \lambda)}{K\left(\omega / \beta^{2}, \beta l, \lambda\right)}$ to be no longer one. This is shown in Fig. 1 where the real and imaginary parts of the ratio $\frac{\beta^{2} K(\omega, l, \lambda)}{K\left(\omega / \beta^{2}, \beta l, \lambda\right)}$ as a function of $\omega l^{2}$ deviate systematically from the constant values 1 and 0 , respectively.

Now it is possible to estimate the slip length $\lambda$ by knowing the value for the ratio $\frac{\beta^{2} K(\omega, l, \lambda)}{K\left(\omega / \beta^{2}, \beta l, \lambda\right)}$. This means we can solve explicitly $\lambda$ as a function of such a ratio and plot curves that might be experimentally used to determine the value of $\lambda$ from the chosen $\omega l^{2}$ and, for example, the real part of the ratio $\frac{\beta^{2} K(\omega ;, /, \lambda)}{K\left(\omega / \beta^{2}, \beta l, \lambda\right)}$. This is shown in Fig. 2.

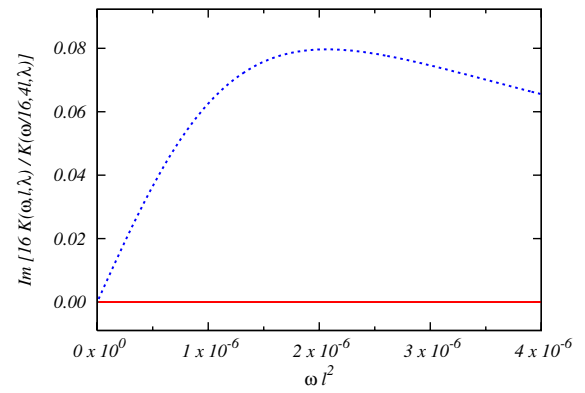

FIG. 1 (color online). Real and imaginary parts of the ratio $\beta^{2} K(\omega, l, \lambda) / K\left(\omega / \beta^{2}, \beta l, \lambda\right)$ vs $\omega l^{2}$ (in $\mathrm{rad} \mathrm{m}^{2} / \mathrm{s}$ ) with $\beta=4, \eta=$ $1.003 \times 10^{-3} \mathrm{~kg} /(\mathrm{ms}), \rho=9.982 \times 10^{2} \mathrm{~kg} / \mathrm{m}^{3}$, and $l=10 \mu \mathrm{m}$ in the absence of slip (continuous line) and for a slip length $\lambda=$ $1 \mu \mathrm{m}$ (dotted line). 


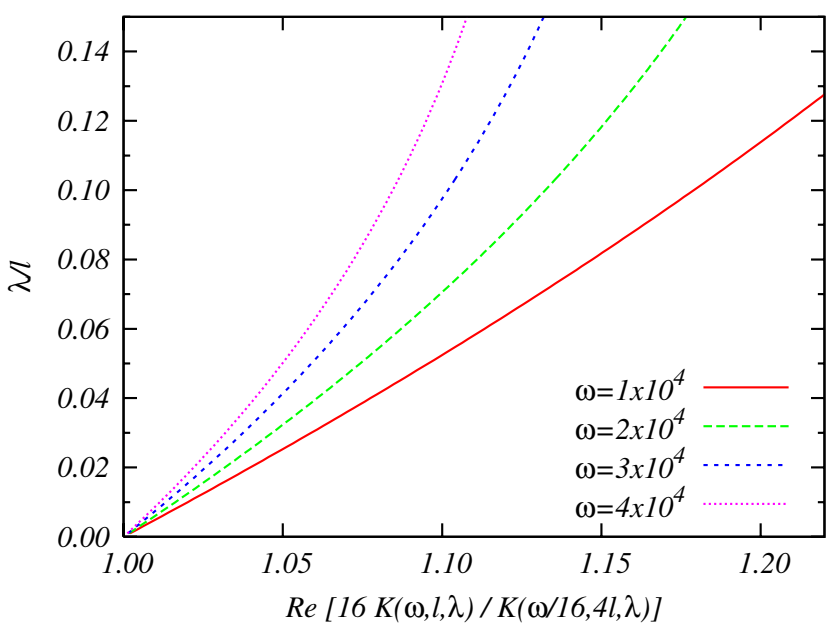

FIG. 2 (color online). Slip length in units of $l$ versus the real part of the ratio $\beta^{2} K(\omega, l, \lambda) / K\left(\omega / \beta^{2}, \beta l, \lambda\right)$ with $\beta=4, \eta=$ $1.003 \times 10^{-3} \mathrm{~kg} /(\mathrm{ms}), \quad \rho=9.982 \times 10^{2} \mathrm{~kg} / \mathrm{m}^{3}, \quad$ and $l=$ $10 \mu \mathrm{m}$ at different values of angular frequency.

If Navier hypothesis holds in dynamic situations (as it is assumed in the present work), any pair of experiments should give exactly the same value of $\lambda$. If the value of $\lambda$ obtained from Fig. 2 for a second pair of experiments [with new values of the real part of the ratio $\frac{\beta^{2} K(\omega ; l, \lambda)}{K\left(\omega / \beta^{2}, \beta l, \lambda\right)}$ and $\left.\omega l^{2}\right]$ were not the same, it would imply that the hypothesis of constant $\lambda$ does not hold in dynamic situations. In other words, we are proposing a way of testing if Navier hypothesis in dynamic situations is valid for a particular system.

In order to experimentally determine the scaling relation like the one shown in Fig. 1 a microparticle image velocimetry ( $\mu$ PIV) could be used [7,25-27]. For our theoretical calculations we have worked with microchannels of 20 and $80 \mu \mathrm{m}$ high and slip lengths of $1 \mu \mathrm{m}$ for water since these are dimensions comparable to the ones used in [7]. We have made a rough estimation of possible uncertainty bars for our scaling relation. Our estimation is based on the experimental precision for velocity of the $\mu$ PIV apparatus

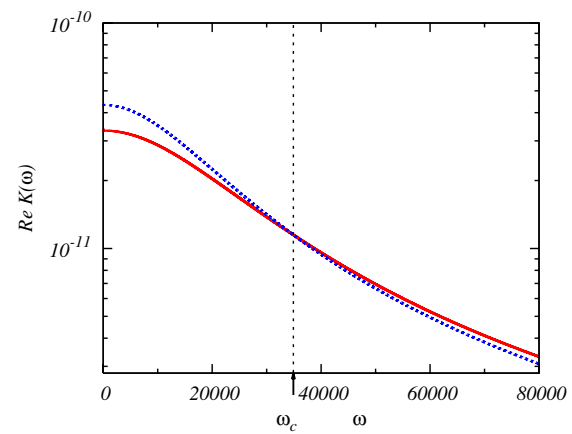

reported in [25] and on the experimental precision for pressure for pressure-driven flows in microchannels [28]. We have obtained a relative uncertainty of $6.4 \%$ for the real part of the scaling relation of Fig. 1 for working pressures of about $1 \mathrm{MPa}$. Since the theoretical difference for the real part of this ratio with and without slip goes from $21 \%$ at low frequencies to $8 \%$ at high frequencies, a particular experiment could choose, in principle, to work with frequencies, in the range between a few hertz and kilohertz, in which the effect would be discernible.

$\mu$ PIV techniques have been used in stationary flows and in oscillatory flows of few tens of $\mathrm{Hz}[27,29]$. Here we present an argument that indicates that $\mu$ PIV techniques can be used at frequencies up to the order of kilohertz. Typical measurements of uncertainty in velocity are governed by uncertainty in displacement. Modern particle tracking can find particle centers to within $10 \%$ of the effective particle diameter [26]. If we wanted a maximum uncertainty in displacement of $2 \%$, we would require displacements of around $5 \mu \mathrm{m}$. This implies that for frequencies of the order of $1 \mathrm{kHz}$, we would require velocities of the order of $5 \mathrm{~mm} / \mathrm{s}$. Modern particle tracking can readily accomplish few $\mathrm{mm} / \mathrm{s}$ [30].

In Fig. 3 we plot the real and imaginary parts of the dynamic permeability [Eq. (5)] as a function of frequency for water in a microchannel. Consistent with results for the steady state known in literature, we obtain that when $\omega \rightarrow$ 0 the stationary permeability of a rectangular microchannel with Navier hypothesis is $K(0, l, \lambda)=\frac{l^{2}}{3}+\lambda l$. This means that slip enhances the flow magnitude of the steady state making smaller the resistance to flow. As Fig. 3 shows, the effect of slip on the permeability depends on frequency. Note that there is a crossing frequency (on the range of sound, $\nu_{c}=\omega_{c} / 2 \pi \sim 5500 \mathrm{~Hz}$ ) of the curves of the real part of the permeability (with and without slip). However, there is no particular signature at this frequency for the imaginary part of the dynamic permeability which, contrary to its zero value in the steady state, has a finite value for an arbitrary frequency. In order to know the flow as a function of time for a particular frequency, both contribu-

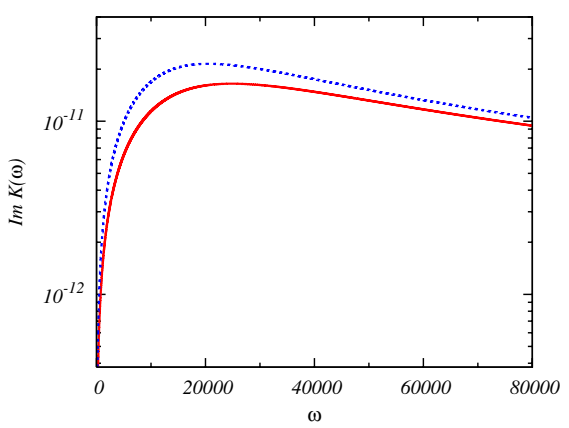

FIG. 3 (color online). Real and imaginary parts of the dynamic permeability (in $\mathrm{m}^{2}$ ) versus angular frequency (in rad/s) with $\eta=$ $1.003 \times 10^{-3} \mathrm{~kg} /(\mathrm{ms}), \rho=9.982 \times 10^{2} \mathrm{~kg} / \mathrm{m}^{3}$, and $l=10 \mu \mathrm{m}$ in the absence of slip (continuous line) and for a slip length $\lambda=$ $1 \mu \mathrm{m}$ (dotted line). 


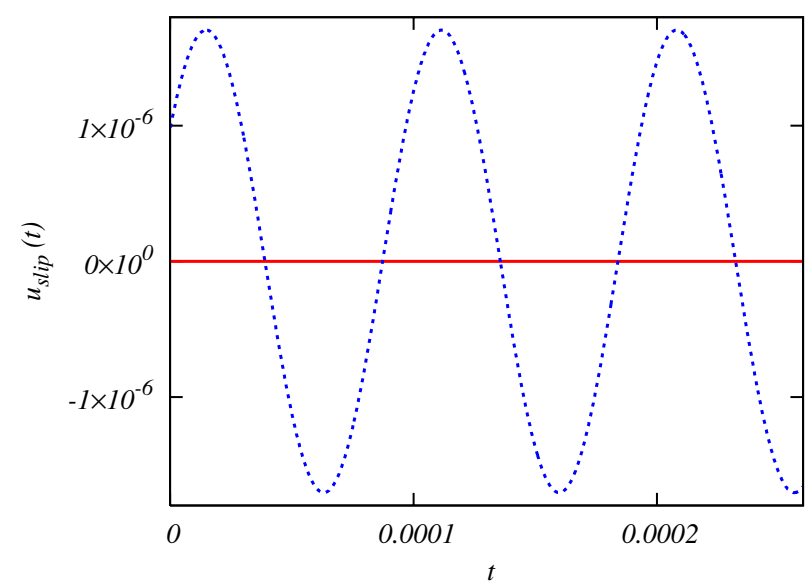

FIG. 4 (color online). Typical behavior of the slip velocity (in $\mathrm{m} / \mathrm{s}$ ) as a function of time (in $\mathrm{s}$ ) for an oscillatory pressure gradient consisting of a cosine wave. Continuous line is for no slip and dotted line corresponds to a slip length $\lambda=1 \mu \mathrm{m}$.

tions should be taken into account as can be seen from Eq. (6). We have computed the flow for a cosine pressure gradient for frequencies before, during, and after the crossing and have found no qualitative difference. In all cases, the magnitude of the flow with slip has been found to be larger than the one with no slip.

Figure 4 shows the slip velocity as a function of time for an oscillatory pressure gradient consisting of a cosine wave with $\omega=65000 \mathrm{rad} / \mathrm{s}$. In order to compute it, we have worked with the local version of the velocity in time domain evaluated at the wall. We have plotted it in the absence of slip and for a slip length of $1 \mu \mathrm{m}$. We can observe that in the case of having a finite slip length, the slip velocity at the wall oscillates in time while for no slip it is always zero.

In conclusion, we have analyzed the dynamical response of Newtonian fluids that obey Navier hypothesis in microchannels subject to nonstationary pressure gradients. In particular, we have analytically computed the dynamic permeability, which contains the information of the fluid response to a dynamic pressure gradient. We have found that in the absence of slip, the dynamic permeabilities of two microchannels obey a scaling relation that is broken when slip is present. This result might be useful to experimentally determine the absence or presence of slip in a system. In addition, if slip exists, this might provide a way to experimentally estimate the slip length $\lambda$, and test the validity of Navier hypothesis in dynamic situations. Our results could be experimentally tested using frequencies of sound and $\mu$ PIV technology since we have worked in the range of parameters relevant in microfluidics [7].

We thank J. G. Santiago and J. L. Arauz-Lara for helpful and interesting discussions. We thank financial support from Dirección General de Investigación (MEC, Spain) through projects FIS2006-12253-C06-05 and FIS200612253-C06-06, DGAPA, UNAM through PAPIIT project
IN101907, and CONACYT (Mexico) through projects 58064 and 83149.

*Author to whom correspondence should be addressed. eugenia.corvera@gmail.com

[1] T. Squires and S. Quake, Rev. Mod. Phys. 77, 977 (2005).

[2] P. Tabeling, Introduction to Microfluidics (Oxford University, New York, 2006).

[3] E. Lauga, M. P. Brenner, and H. A. Stone, in Handbook of Experimental Fluid Dynamics, edited by C. Tropea, A. Yarin, and J. F. Foss (Springer, Berlin, 2007), Chap. 19.

[4] C. Cottin-Bizonne, J.-L. Barrat, L. Bocquet, and E. Charlaix, Nature Mater. 2, 238 (2003).

[5] G. Whitesides, Nature (London) 442, 368 (2006).

[6] J. Ottino and S. Wiggins, Science 305, 485 (2004).

[7] D. Tretheway and C. Meinhart, Phys. Fluids 14, L9 (2002).

[8] V. Craig, C. Neto, and D. Williams, Phys. Rev. Lett. 87, 054504 (2001).

[9] C.-H. Choi, K. J. A. Westin, and K. S. Breuer, Phys. Fluids 15, 2897 (2003).

[10] J. Koplik and J. R. Banavar, Phys. Rev. Lett. 96, 044505 (2006).

[11] S. Granick, Y. Zhu, and H. Lee, Nature Mater. 2, 221 (2003).

[12] Not always so for polymer melts [13].

[13] W. Schowalter, J. Non-Newtonian Fluid Mech. 29, 25 (1988).

[14] P. Thompson and S. Troian, Nature (London) 389, 360 (1997).

[15] Y. Zhu and S. Granick, Phys. Rev. Lett. 87, 096104 (2001).

[16] E. Lauga and M. Brenner, Phys. Rev. E 70, 026311 (2004).

[17] P. de Gennes, Langmuir 18, 3413 (2002).

[18] C. Neto, D. Evans, E. Bonaccurso, H. Butt, and V. Craig, Rep. Prog. Phys. 68, 2859 (2005).

[19] E. Lauga and H. Stone, J. Fluid Mech. 489, 55 (2003).

[20] P. Joseph and P. Tabeling, Phys. Rev. E 71, 035303 (2005).

[21] M. López de Haro, J. A. del Río, and S. Whitaker, Phys. Rev. E 58, 6323 (1998).

[22] E. Corvera Poiré and J. A. del Río, J. Phys. Condens. Matter 16, S2055 (2004).

[23] R. Collepardo-Guevara and E. Corvera Poiré, Phys. Rev. E 76, 026301 (2007).

[24] Local versions of Eqs. (4)-(6) can be easily written if one wishes to experimentally determine the velocity at only one plane of the microchannel (for example the middle plane with $z=0$ ).

[25] J. G. Santiago, S. T. Wereley, C. D. Meinhart, D. J. Beebe, and R. J. Adrian, Exp. Fluids 25, 316 (1998).

[26] C. Meinhart, S. Wereley, and J. Santiago, Exp. Fluids 27, 414 (1999).

[27] S. Devasenathipathy, J. Santiago, S. Wereley, C. Meinhart, and K. Takehara, Exp. Fluids 34, 504 (2003).

[28] Chang-Hwan Choi, K. Johan A. Westin, and Kenneth S. Breuer, Exp. Fluids 34, 635 (2003).

[29] M. Oddy and J. Santiago, J. Colloid Interface Sci. 269, 192 (2004).

[30] J. G. Santiago (private communication). 